\title{
REMOTE FIELD-PROGRAMMABLE GATE ARRAY (FPGA) LAB
}

\author{
Karthik.S ${ }^{1}$, P.Shreya ${ }^{2}$, Srihari $\mathbf{P}^{3}$, N.M.Viswanath ${ }^{4}$ \\ ${ }^{1}$ Assistant professor, Department of ECE, SRM University Vadapalani, TN, India \\ ${ }^{2}$ Student, Department of ECE, SRM University Vadapalani, TN, India \\ ${ }^{3}$ Student, Department of ECE, SRM University Vadapalani, TN, India \\ ${ }^{4}$ Student, Department of ECE, SRM University Vadapalani, TN, India
}

\begin{abstract}
In today's world, e-learning has proved to be a very useful technology. In addition to e-learning, e-labs are also being implemented. E-labs can be either virtual or remote. This paper is an initiative to create a remote fpga lab. The implementation of the same is also complete. The base kit used is the spartan $3 E$ family of FPGA which is interfaced with an arduino board through a LabView interface for providing the input into the FPGA from the remote desktop. The outputs are displayed in the remote computer. The outputs can also be viewed through the webcam. The main intention of this project is to make distance learning easy. It is also an advantage for students as additional experiments that do not come under the syllabus can be performed anytime, anywhere.
\end{abstract}

Keywords:-Spartan3E, Arduino, Labview etc.

\section{INTRODUCTION}

Today, the use of remote labs for distance learning in the electronics field is increasing at a rapid rate. Unlike virtual labs, which provide software simulations of physical processes, remote labs allow users to remotely work on real experiments, thus making it more realistic and test its performance. Experimental testing is crucial in the field of VLSI, where the validation real data is extremely important for assessing the effectiveness of the design. However, performing such experiments is both difficult and expensive, particularly when handling complex FPGA kits such as virtex or spartan. Therefore, the chance performing every experiment individually may be difficult for all the students, especially in case of large classes This paper presents a remote fpga lab, based on the XLINX software, Spartan-3E FPGA (XC3S500E) and LabVIEW software. XILINX represents a standard tool in VLSI design education because of its easy-tolearn and powerful language. Using XILINX as the main software tool has several advantages. It provides both a friendly environment for the students to interact with the remote lab as well as a programming language that they are likely to be familiar with. Meanwhile, it makes the interaction with the lab independent of chosen circuit design and its lowlevel programming language, which is often a barrier for entry-level users. Apart from the Xilinx software, LabVIEW is used to setup an interface [7] between the remote desktop and the FPGA kit. LabVIEW is commonly used for data acquisition, instrument control, and industrial automation on a variety of platforms including Microsoft Windows, various versions of UNIX, Linux, and Mac OS X, for its simplicity and low cost.The remote lab has been implemented and is in its early stage. Once set up, the main goal of this lab is to permit students to execute their theoretical knowledge at a practical level in concepts like counters and multipliers in a simple way, without any restraints due to laboratory session timings or availability of hardware components. This paper is structured as follows. In the following section, Section 2, the proposed remote FPGA lab, along with its hardware architecture, is described in detail. Section 3 deals with the software architecture. A typical user session is presented in Section 4, while in Section 5 some conclusions are drawn.

\section{HARDWARE ARCHITECTURE}

This section deals with the three main hardware used in this project viz. the Spartan 3E FPGA starter kit, Arduino Uno board, and a core2duo intel processor PC

\subsection{FPGA [1]}

The spartan 3E family of Field Programmable Gate Array (FPGAs) is specifically designed to meet the high volume needs, and its cost sensitive consumer electronic application gives it the much required edge over the other versions. Because of their exceptionally low cost the SPARTAN 3E FPGAs are ideally suited to a wide range of consumer electronics application including broadband access, home networking, display/projection etc.The spartan 3E starter kit uses FPGAs programmed by loading configuration data into robust reprogrammable, static CMOS configuration latches (CCLs) that collectively control all functional elements and routing resources. The FPGA's configuration data is stored in a PROM or some other non-volatile medium, either on or off the board. The evaluation board of the starter kit contains several embedded peripheral modules. These may include 8 LED indicators, facility to input 8bit umbers using Toggle switches, stepper motor interface with on-board motor, DC 
motor interface with on-board motor supporting direction control, audible buzzer indicator, UART serial port interface etc. The wide range of peripherals provided by XC3S250E offers many interfacing options which enables the user to study, analyze and implement programs.

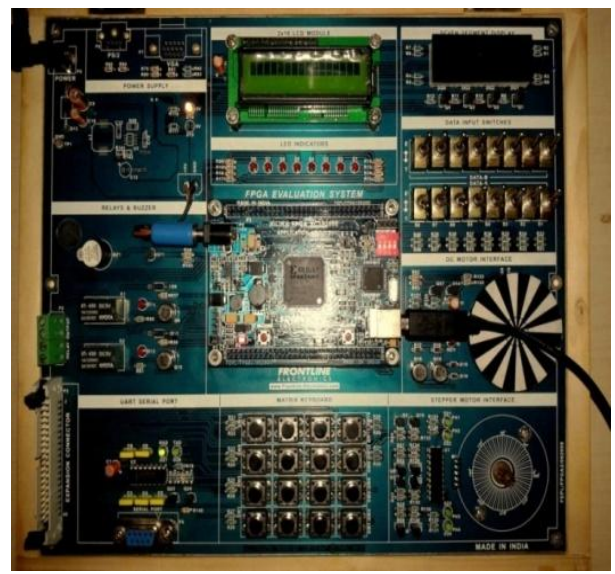

Fig-1 Spartan 3E kit

\subsection{Arduino Uno Board}

The Arduino Uno [4] is a microcontroller board based on the ATmega328. It has 14 digital input/output pins, 6 analog inputs, a $16 \mathrm{MHz}$ ceramic resonator, a USB connection, a power jack, an ICSP header, and a reset button. It contains everything needed to support the microcontroller. It can be connected to a computer with a USB cable or powered with a AC-to-DC adapter or battery to get started. The Uno differs from all preceding boards in that it does not use the FTDI USB-to-serial driver chip. Instead, it features the Atmega16U2 programmed as a USB-to-serial converter. The Arduino acts as the interface between the computer and the FPGA kit, in order to transfer the binary data that is generated by the user in the computer as input for the experiment. Since the ATmega328 has the capacity to send and receive binary data, it is used to view the output on the graphical user interface (GUI) in the LabVIEW software.

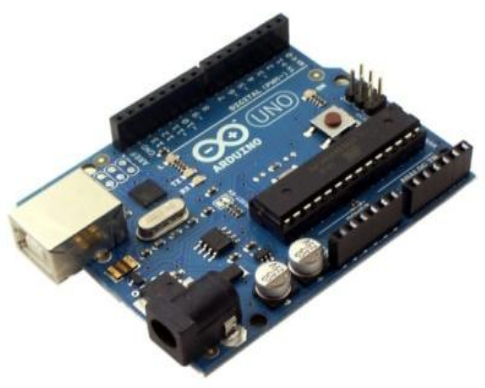

Fig-2 Arduino

\subsection{PC Server}

A desktop Personal Computer (PC) acts as the core of the project, providing the necessary processing power. The Xilinx software runs in background to perform all computations. The $\mathrm{PC}$ is connected to the Internet to accept connection to the web server it hosts. Through an Arduino board the PC communicates with the FPGA. Moreover, it is connected to a webcam for online video streaming.

\section{SOFTWARE ARCHITECTURE}

Xilinx XC3S250E FPGA uses Xilinx ISE software for program implementation. The user is expected to know the basics of Xilinx for implementing HDL programs. The Xilinx software can be downloaded from the Xilinx website [3]. The application program is in XSVF format which can be configured into FPGA or programmed into data flash. Top view programmer-Xilinx FPGA software is used for configuring the program.

LabVIEW [8] is a system-design platform and development environment for a visual programming language from National Instruments. Dataflow programming language is used. The user connects different function-nodes by drawing wires in a graphical lock diagram. Parallel execution is also possible. Labview also uses graphical programming. It basically has 3 components: connector panel, front panel, block diagram. Front panel is built using inputs and outputs. Connector panel is used to represent programs in other programs. The block diagram also known as the back panel contains the graphical source code. Hence lab equipments can be virtually represented using labview. Hence inputs from the remote computer to the equipment in the lab can be given easily. The outputs can also be viewed similarly.

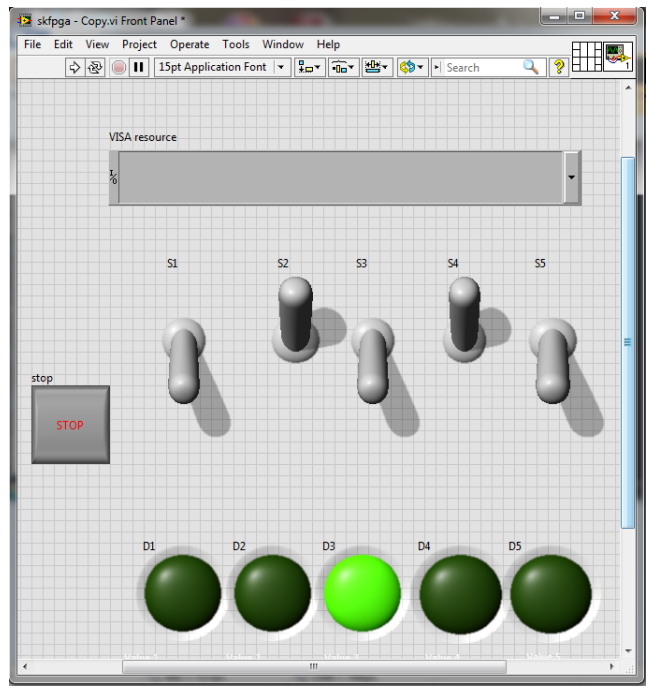

Fig-3 Labview interface 


\section{SESSION DESCRIPTION}

This section illustrates an overview of a typical user session.The user initially connects to the given webpage through a common browser in which all the tools like Xilinx, labview etc. are all integrated. Students who are using the Elabs for the first time have to register with the website thus creating an account for them. Once they $\log$ in to their accounts they choose the Xilinx ISE platform and enter the program they wish to execute. ISE design tool "impact" is used for implementation. Once the program is implemented it is mounted on to the data flash of the FPGA kit by the student from the remote desktop. Thus the FPGA is successfully configured.

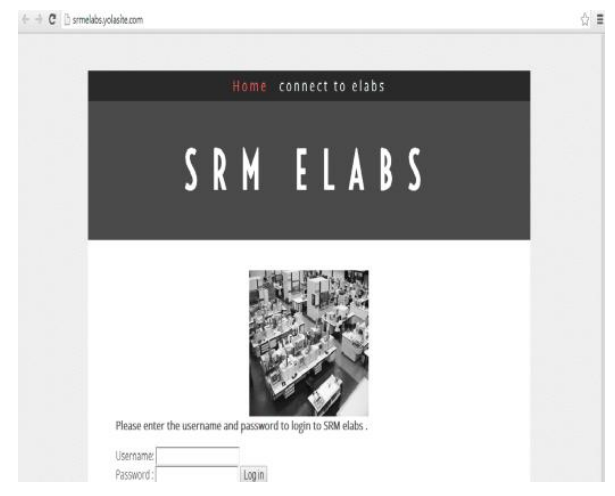

Fig-4 Login Environment

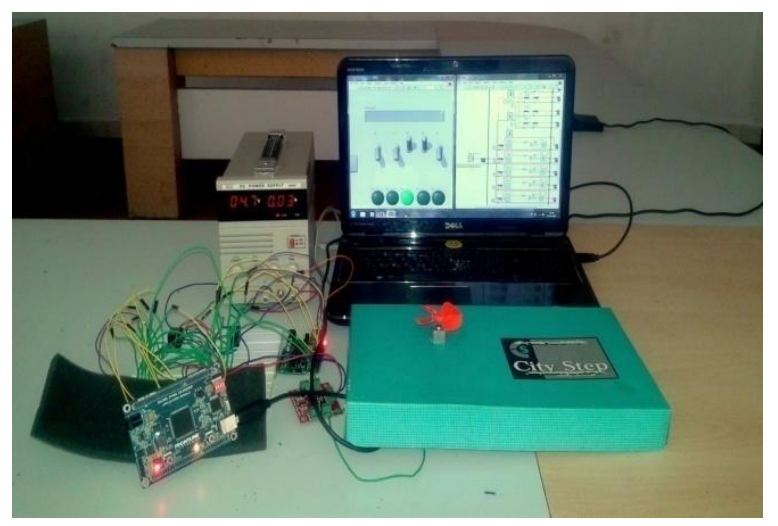

Fig-5 Remote Lab Setup

However, in order to interface the input and output of the FPGA kit with the PC, LabVIEW software is used.To connect the PC with the FPGA an ARDUINO-UNO board is used. The webpage contains the link to the LabVIEW software where the inputs have to be given. These inputs are transferred to the FPGA kit through the ARUIDNO board. The corresponding output from the FPGA is sent to the ARDUINO which is then viewed on the PC screen. A webcam is also interfaced with the computer. Therefore the students can view the outputs through the webcam.

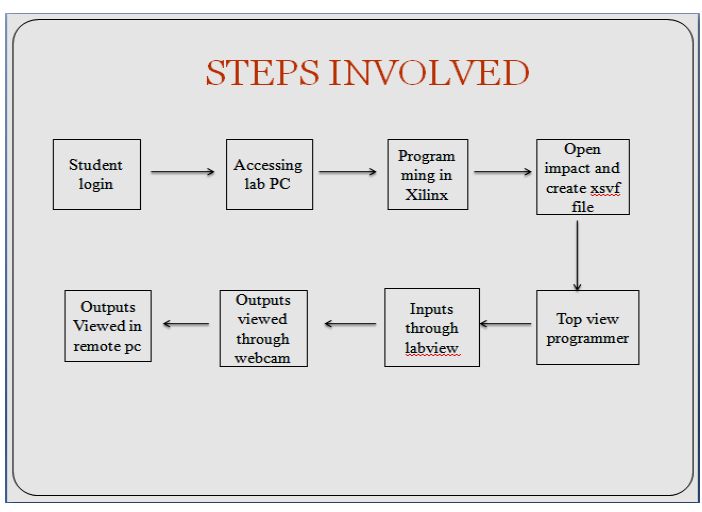

Fig-5 Steps involved

\section{ACKNOWLEDGMENTS}

We would like to thank all the faculties and friends of SRM University who helped us in completing our project. We would also like to thank our university for providing us with the necessary facilities and arrangements.

\section{CONCLUSIONS}

In this paper, a remote lab for implementing HDL designs in FPGA kits has been presented. Through an Internet connection, a user can implement the design using Xilinx ISE. A graphical interface, along with video streaming provides the user an input-output interface. Thanks to the low-cost setup which features high versatility, it is possible to test, analyze and implement complex circuit design. The lab is made available 24 hours a day, so that students can experiment various concepts remotely. With the enlargement of the experimental area, it is also expected to increase the number of FPGA setup, in order to execute programs on a multiple user basis. In the future, it is planned to enrich the user interface with additional features

\section{REFERENCES}

[1]. FPGA evaluation manual.

[2]. www.origin.xilinx.com/fpga

[3]. www.xilinx.com > Products > Development Tools > ISE Design Suite-ise web pack

[4]. en.wikipedia.org/wiki/Arduino-arduino

[5]. http://www.ni.com/labview/-about labview

[6]. http://www.ni.com/pdf/manuals/320999b-lab view manual [7]. http://vishots.com/getting-started-with-the-labviewinterface-for-arduino/-labview arduino interface

[8]. en.wikipedia.org/wiki/LabVIEW-about labview

[9]. Morgan, F.; Cawley, S. "Enhancing learning of digital systems using a remote FPGA lab "published in Reconfigurable Communication-centric Systems-on-Chip (ReCoSoC), 2011 6th International workshop.

[10]. El Medany, W.M., Al Fayyum "FPGA remote laboratory for hardware e-learning courses" published in Computational Technologies in Electrical and Morgan, F.; Cawley, S. 
"Enhancing learning of digital systems using a remote FPGA lab "published in Reconfigurable Communication-centric Systems-on-Chip (ReCoSoC), 2011 6th International Workshop.

\section{BIOGRAPHIES}

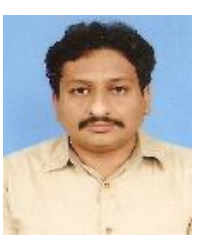

Karthik $\mathbf{S}$ was born in Chennai, in 1981. He received the B.E degree from University of Madras, Chennai, in 2003 and M.Tech degree from Sathyabama University, Chennai in 2005 and also working towards the PhD. Degree in area of Hidg Performance Computing.He works as an ASSISTANT PROFESSOR at SRM University, Vadapalani and his area of interest includes, dynamic partial reconfiguration, low power circuits and VLSI verification and testing.

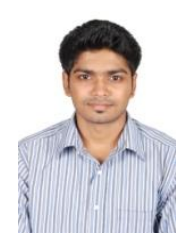

Mr.N.M.Viswanath is currently pursuing B.Tech ECE at SRM University. He has a passion for electronics and is always abreast with the current technological

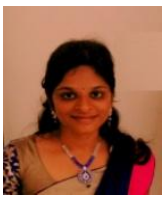

Ms.P.Shreya is pursuing her bachelor's degree in ECE at SRM University. Her areas of interest are VLSI and ASIC. She is very keen in learning new things.

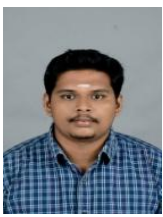

Mr.Srihari $\mathrm{P}$ is pursuing his B.Tech degree in ECE at SRM University. His areas of interest are VLSI and digital systems and is keen in working on new concepts. 